\title{
TTR
}

Traduction, terminologie, re?daction

\section{En anglais, Léonora parle-t-elle encore et à qui?}

\section{Anne Malena}

Volume 10, numéro 1, 1er semestre 1997

Langues, traduction et post-colonialisme

Languages, Translation and Post-Colonialism

URI : https://id.erudit.org/iderudit/037284ar

DOI : https://doi.org/10.7202/037284ar

Aller au sommaire du numéro

\section{Éditeur(s)}

Association canadienne de traductologie

ISSN

0835-8443 (imprimé)

1708-2188 (numérique)

Découvrir la revue

Citer cet article

Malena, A. (1997). En anglais, Léonora parle-t-elle encore et à qui? TTR, 10(1), 197-217. https://doi.org/10.7202/037284ar

\section{Résumé de l'article}

En anglais, Leonora parle-t-elle encore et à qui? — Dany Bébel-Gisler, sociolinguiste et attachée de recherche au C.N.R.S., a écrit Leonora : l'histoire enfouie de la Guadeloupe afin de célébrer la culture de son île natale et de produire un contre-discours au discours national français. À partir d'une série d'entrevues en créole avec Léonora, travailleuse de canne née en 1919, Dany établit le texte d'un roman-témoignage dans lequel elle maintient certains passages en créole. Bien que Bébel-Gisler s'approprie la voix de Léonora, elle le fait dans un but didactique et militant et cet acte littéraire de traduction peut être défendu comme un geste de solidarité visant à explorer et à faire connaître le pouvoir de résistance des pratiques culturelles de la Guadeloupe. Le présent article analyse la version américaine de Léonora traduite par Andrea Leskes, publiée par " University Press of Virginia ", afin de déterminer si elle parvient à renouveler ce geste de solidarité. Une question bakhtinienne (Qui parle?) est posée et trois problèmes spécifiques sont examinés pour tenter d'y répondre : l'emploi du créole, la relation dialogique entre le créole et le français et la valeur de contre-discours du roman.
Tous droits réservés (C) TTR: traduction, terminologie, rédaction — Les auteurs, 1997
Ce document est protégé par la loi sur le droit d'auteur. L'utilisation des services d'Érudit (y compris la reproduction) est assujettie à sa politique d'utilisation que vous pouvez consulter en ligne.

https://apropos.erudit.org/fr/usagers/politique-dutilisation/ 


\section{En anglais, Léonora parle-t-elle encore et à qui?}

\section{Anne Malena}

\section{Introduction}

En tant qu'acte de langage, la traduction superpose un deuxième rapport discursif à celui, déjà complexe, qui reliait le texte original à son premier public. Dans le cas d'une cuvre provenant d'une culture marginalisée, cette relation s'avère d'une telle complexité qu'il est nécessaire de se poser de multiples questions pour commencer à en comprendre le fonctionnement et les ramifications possibles. Il faut, par exemple, s'interroger sur les relations historiques entre la langue de départ et la langue d'arrivée, sur les différentes motivations qui ont d'abord produit l'original et ensuite la traduction, sur les similarités et les différences entre les deux maisons d'édition, sur la définition des publics visés et sur la place occupée par ce texte dans les deux systèmes littéraires. L'étude qui suit ne prétend pas répondre en détail à toutes ces questions mais propose une lecture de la traduction américaine d'un texte francophone peu connu, dans l'espoir de contribuer à élucider les enjeux de l'entreprise "postcoloniale" de traduction'.

\footnotetext{
' Le terme opostcolonialı demande à être placé entre guillemets ici parce que, dans le cas qui nous occupe, le texte étudié est issu d'une région (la Guadeloupe) qui continue à être administrée par l'ancien pouvoir colonial. Pour un traitement
} 


\section{Traduire la francophonie}

La collection Caraf Books, créee par les éditions «University Press of Virginiaw, publie en traduction les chefs-d'cuvre des régions francophones et mérite d'être applaudie pour ses efforts de transmission de savoirs culturels. En plus du livre dont il est question ici, Leonora : The Buried Story of Guadeloupe de Dany Bébel-Gisler, ont déjà paru dans cette collection des titres provenant de l'Afrique de l'Ouest, du Maghreb et des Antilles ${ }^{2}$. La notice explicative à la fin de chaque livre rend évidentes les intentions didactiques de la collection et la liste de ses éditeurs (James Amold, Kandioura Dramé, Michael Dash et Henry Louis Gates) en souligne la teneur intellectuelle. En ma qualité de traductrice, je souhaite également reconnaître la difficulté et la valeur du travail entrepris par Andrea Leskes, traductrice de Léonora, indépendamment des questions que je soulève sur le devenir de la voix et de l'identité de Léonora.

\section{Qui parle?}

Léonora, travailleuse de canne née en Guadeloupe en 1919, a accordé une série d'entrevues à la sociologue Dany Bébel-Gisler vers la fin des années 1970 et au début des années 1980. De cette collaboration est issu un livre, Léonora: l'histoire enfouie de la Guadeloupe (1985a), dans lequel Dany Bébel-Gisler propose de célébrer la culture de son île natale et de produire un contre-discours au discours national français. Pour ce faire, elle traduit en français les entrevues données en créole par Léonora et transforme ces entrevues en chapitres dont les titres sont fournis par les paroles de Léonora et les épigraphes par des citations de chercheurs, d'écrivains et de théoriciens. Elle établit ainsi le texte d'un roman-témoignage, ou d'une biomythographie, selon le terme de Audre Lorde (Boyce et Savory, 1990,

suggestif du potentiel de la traduction en tant que force de résistance et de transformation dans un contexte postcolonial, voir Niranjana.

2 Voir, en particulier, Bhêly-Quénum, Fantouré, Oyơno̊-Mbia et Badian, U Tam'Si, Djebar, Kateb, Césaire, Condé, Depestre, Glissant, Juminer, Maximin et Senghor. 
p. 5), dans laquelle elle maintient certains passages en créole ${ }^{3}$. Elle ajoute un épilogue justifiant son projet et des notes expliquant les références culturelles et historiques. Bien que Bébel-Gisler s'approprie la voix de Léonora, elle le fait dans un but didactique et militant, et cet acte littéraire de traduction peut être défendu comme un geste de solidarité visant à explorer et à faire connaître les pratiques culturelles de la Guadeloupe. Dany Bébel-Gisler, elle-même Guadeloupéenne, milite depuis de longues années pour l'alphabétisation des adultes en créole, pour l'enseignement du créole dans les écoles et pour un système scolaire qui reconnaisse le besoin des Enfants de la Guadeloupe, pour reprendre le titre d'un de ses nombreux ouvrages critiques (1985b), de s'identifier à une culture et à une langue propres.

Dans le Département d'Outre-Mer (DOM) qu'est la Guadeloupe, il n'y a pas si longtemps, on pouvait encore entendre les enfants d'anciens esclaves, assis sur les bancs d'école, ânonner "Nos ancêtres les Gaulois...". Léonora en parle, de cette expérience humiliante de l'école où il est interdit, sous peine de se faire battre, de parler sa langue maternelle et où, par nécessité, les enfants apprennent par cœur les quelques formules françaises qui leur paraissent les plus importantes, sans toujours les comprendre d'ailleurs ${ }^{4}$. Léonora a été l'une de ces malheureuses gamines et le livre que Dany conçoit, à partir de ses paroles, est une ceuvre de restitution de son identité créole et guadeloupéenne. Dany a traduit Léonora afin de la présenter comme une figure de résistance à la domination française : la langue créole parlée par Léonora est un véhicule de révolution, position théorique que Dany Bébel-Gisler avait déjà

${ }^{3}$ A propos du rapprochement de Léonora au genre hispanique du testimonio, voir Amold ainsi que Kutzinski et Mesh-Ferguson; pour plus de détails sur la narration de Léonora, voir Malena. Je tiens aussi à remercier Cynthia Mesh-Ferguson d'avoir eu l'amabilité de me laisser consulter deux travaux inédits sur Léonora.

${ }^{4}$ Léonora commente les distorsions humoristiques, et révélatrices, du pouvoir subversif du créole, que les chants latins, faute de compréhension, subissaient à l'église :

Quant aux chants en latin, on les peignait en créole : Ave Maria devenait lavé lari la (lavez la rue). laudate, dlo glasé (de l'eau glacée); miséricorde, miziré kòd (mesurez la corde), etc. (p. 247-248). 
exposée dans La langue créole, force jugulée (1976). Travailleuse de canne, femme de contremaître, participante aux grèves des années 1970 et militante pour l'adoption du créole à l'église, Léonora illustre, par le récit de sa vie, le combat inlassable que sa communauté livre pour améliorer les conditions socio-économiques qui lui sont imposées depuis des siècles ${ }^{5}$. Dans une perspective postcoloniale, la question se pose de savoir si la version américaine de Léonora parvient à renouveler le geste solidaire accompli par Dany ou si, au contraire, elle le fausse ou l'entrave.

Selon les théories de l'énonciation, le destinataire d'un acte de langage en devient le co-énonciateur. La traductrice, de son côté, se pose à la fois comme destinataire et destinatrice de ce même acte de langage ${ }^{6}$. Dans le cas de Léonora, le texte établi par Dany est déjà une transposition du dialogue non itératif qui a pris place entre les deux femmes. La traduction anglaise détourne l'énonciation originale en fonction d'une nouvelle destinatrice (la traductrice) et d'un nouveau destinataire (le lectorat américain). Mon analyse de la traduction et de ses conditions de publication cherche à déterminer quels changements les paroles de Léonora subissent en passant des langues de départ (le créole et le français) à la langue d'arrivée (l'anglais) à travers les étapes successives d'écoute, d'écriture, de lecture et de traduction. Mon étude pose une question bakhtinienne (Qui parle?) ${ }^{7}$ et examine le traitement de trois problèmes spécifiques dans la langue d'arrivée pour tenter d'y répondre : l'emploi du créole, la relation dialogique entre le créole et le français et la valeur de contre-discours du roman.

${ }^{5}$ Voir le chapitre que j'ai consacré a Léonora dans The Dynamics of Identity in Francophone Caribbean Narrative, à paraître chez Peter Lang.

' Pour les théories de l'énonciation, voir Cervoni, Charaudeau, Danon-Boileau, Kerbrat-Orecchioni et Ducrot; pour la traduction en tant que ré-énonciation, voir Folkart.

- Bien que la question «Qui parie?» fasse écho à celle de Gérard Genette qui l'utilise en conjonction avec celle de "Qui voit?’, mon analyse se place plutôt dans une perspective informé par les théories de Michael Bakhtine sur la polyphonie. 


\section{Un déplacement subtil}

En ce qui conceme les passages en créole, ils ne posent apparemment aucun problème autre que le changement évident de la relation bilingue, puisqu'ils sont reproduits intacts dans la version anglaise. Pourtant, une différence typographique qui s'annonce dès le prologue remet en question certaines décisions éditoriales. Il s'agit de l'emploi des italiques qui, dans l'original, sont l'objet d'un jeu subtil de juxtaposition alors que, dans la traduction, ils sont strictement réservés aux mots et aux courtes expressions créoles qui, à leur tour, font l'objet d'un glossaire dont il sera question plus bas. Ainsi, un rapport visuel d'égalité est créé entre le créole et l'anglais au cours des longs passages qui, en français, portaient la marque de l'auteure. Par exemple, sous l'effet de la traduction, quatre glissements typographiques subtils effacent la marque de la première traductrice, Bébel-Gisler, d'un poème qui précède le premier chapitre : le titre du poème en devient le premier vers, les italiques disparaissent, le nom de Bébel-Gisler devient superflu et le nom de Sonny Rupaire est déplacé au bas de la version traduite du poème (voir appendice, exemple 1).

Dans le prologue, que ce poème vient conclure et dont la voix narrative est partagée entre Dany Bébel-Gisler, Anmann, paysanne guadeloupéenne de quatre-vingt-dix-huit ans et le poète Sonny Rupaire, l'emploi des italiques servait à marquer l'intervention de l'auteure traductrice et sa manipulation des propos recueillis. De cette façon, le contraste entre les types romain et italique permettait aussi de mettre en évidence le rapport d'altérité de la langue créole à la langue française, rapport aisément oublié à cause de la proximité phonologique des deux langues, et de reconnaître aux énoncés créoles, dans le contexte du livre, un statut privilégié. L'emploi des italiques vient en effet dans la suite du récit rappeler à la lectrice dans chaque titre de chapitre et dans chaque passage en créole que c'est Léonora qui parle.

Sans ce marquage typographique, la situation d'énonciation apparaît simplifiée, impression renforcée par d'autres détails éditoriaux : les notes en bas de page, qui fournissaient une traduction immédiate des termes créoles retenus dans le texte, sont reléguées au glossaire ou aux notes de fin de volume. Avec l'ajout nécessaire des notes de la traductrice, le nombre des notes de fin de volume passe ainsi de 46 
à 98. Ce changement quantitatif, banal en soi, révèle une transformation plus profonde. Là où les notes de Dany Bébel-Gisler constituaient un apport socio-historique clairement maintenu comme paratexte, les notes du texte anglais s'avèrent diffuses et, avec le glossaire, provoquent une lecture différente. On serait tenté de lire le récit en évitant de se référer aux notes, choix de lecture qui produit des résultats différents en anglais et en français : dans le second cas, on viendrait à manquer d'un certain bagage encyclopédique et, partant, du discours critique de la sociologue au sujet des paroles de Léonora. En anglais par contre, en la triple absence des notes métalinguistiques de bas de page, de celles de la traductrice et de l'annotation critique, les termes créoles et les références obscures risquent de colorer d'exotisme les propos de Léonora. Ce risque d'ailleurs n'est pas totalement effacé par une lecture attentive et docile lorsque le lecteur est contraint, comme le remarque Janet Paterson a propos de Trou de mémoire (1993, p. 48), de passer "d'un espace textuel à un autre et par là d'un code de lecture à un autrem. En fonction de son destinataire américain, la voix de Léonora est ainsi laissée à elle-même et reste enfermée dans son altérité, ou accompagnée d'une annotation érudite qui modifie ses conditions d'énonciation.

\section{De quelle histoire s'agit-il?}

Dany Bébel-Gisler voulait nous faire entendre l'histoire de Léonora afin de mieux nous faire saisir le lieu historique, géographique, politique et personnel d'où elle parle. Elle termine en effet le livre par un épilogue qui s'achève sur les mots suivants (1985a, p. 299) : «Du fond de cette terre, une fermme, une paysanne, prend la parole pour clamer sa différence et ses contradictions, celles de tout un peuple. Un peuple assoiffé d'avenir humain, de justice, d'indépendance et de dignités. En élevant Léonora au rôle de porte-parole du peuple guadeloupéen, Dany transforme ses paroles en un contre-discours et insiste sur l'importance historique d'un récit féminin, par contraste avec la version officielle de l'Histoire. En inscrivant sa propre subjectivité dans le texte, elle présente le témoignage d'une compatriote comme une cuvre littéraire qui autorise la superposition des voix et qui refuse de trancher entre les genres. La traduction du sous-titre L'histoire enfouie de la Guadeloupe par The Buried Story of Guadeloupe relègue la dimension historique du récit au niveau de l'implicite et en privilégie le côté fictionnel par le choix du mot story. De plus, l'indication $A$ novel inscrite en rouge sur la couverture, 
précision nullement rendue explicite dans le texte original, tranche l'ambiguîté des genres sur laquelle jouait le texte français, et présente le texte anglais comme un texte traduit, rangé et édulcoré.

Cette tendance se confirme par d'autres détails qu'il vaut la peine de passer en revue. La première note en fin de volume est une note de la traductrice et fait part de sa décision de traduire le mot Nègre, relativement fréquent, par black, black folks ou blacks parce que, dit-elle, la traduction de ce terme "poses particular problems and touches on culturally sensitive issues» (1994, p. 237). Je ne nie pas les difficultés associées au transfert culturel de ce terme mais je regrette l'effet neutralisant de ce choix uniforme. Il y aurait eu lieu, me semble-t-il, de nuancer la traduction selon le contexte. La complexité de la situation d'énonciation dans laquelle s'inscrit le récit de Léonora exige de reconnaître le caractère polyphonique, selon le terme bakhtinien, de sa voix. En se servant des analyses de Ducrot, on s'aperçoit que la prise de parole de Léonora constitue un «événement énonciatif" dont l'origine est multiple (1984, p. 179). Dany met en effet en scène le discours de Léonora qui, elle-même, est à la fois sujet parlant, pour Ducrot «un élément de l'expériencen, et locutrice, ou ufiction discursive (1984, pp. 198-199). Toujours selon Ducrot, Léonora est locutrice en tant que telle et locutrice wen tant qu'être du mondew $(1984$, p. 199). Ainsi, la vieille dame qui entre en dialogue avec Dany est un être empirique, le sujet parlant, et la narratrice qui nous est donnée à lire est un être de parole.

Ce décorticage des voix multiples dont se compose la parole de Léonora permet de souligner la valeur collective et politique de cette parole, valeur qui disparait en anglais parce que les nuances polyphoniques ne sont pas respectées. Léonora, la «locutrice en tant que telle», utilise souvent le mot créole Nèg pour désigner ses semblables mais, puisqu'elle est aussi «être du monde", c'est-à-dire être historique, il est impossible de lui assigner la responsabilité totale de l'usage de ce terme. Léonora, traduite par Dany, dit Nègre parce que ce terme se réfère à sa réalité quotidienne et marque le contraste de son monde par rapport à la France dont la réalité s'insinue dans sa vie quotidienne. L'exemple suivant, tiré d'un chapitre traitant de son expérience scolaire, illustre bien que Négresse est une notion assimilée dans le discours, et qui ne mérite donc pas les guillemets réservés à bébés et à matrones : 
Une de nos amies, enfant gâtée, se faisait acheter par sa mère des poupées venues de France, aux beaux cheveux et aux yeux bleus. C'étaient nos *bébésw. Nos Négresses de chiffon étaient «matronesw. Elles representaient les grand-mères (1985a, p. 45).

One of our friends, a spoiled child, made her mother buy dolls from France, dolls with beautiful hair and blue eyes. They were our ababies.» Our black rag dolls were matrons. They represented the grand-mothers (1994, p. 31).

Ce passage fait subtilement état d'un double processus de représentation du langage opéré premièrement dans la narration de Léonora et deuxièmement dans la traduction de Dany. Par le truchement du récit autobiographique, Léonora se rend compte de l'assimilation des stéréotypes et de l'imposition du discours idéologique français sur ses énoncés et ses jeux enfantins; Dany, par la traduction et par la mise en scène de Léonora en tant que personnage, rend explicite cette prise de conscience et souligne l'hybridisation de l'énoncé en ayant soin d'encadrer bébés et matrones par des guillemets et de retenir le mot négresse. Bakhtine explique comment l'art littéraire du roman permet de passer de «l'hybridisation involontaire" à «l'hybride intentionnel et conscient" où

se mélangent non pas deux consciences linguistiques impersonnelles (correlats de deux langages), mais deux consciences linguistiques individualisées (corrélats de deux énoncés, non de deux langages) et deux volontés linguistiques individuelles : la conscience et la volonté individuelles de l'auteur qui représente, et la conscience et la volonté individualisées d'un personnage représenté (1978, p. 176).

La perte, dans la version anglaise, des guillemets marquant la valeur idéologique de matrons et de la marque créole du mot négresse, atténue la richesse dialogique de ce passage en le réduisant à la dimension anodine d'une anecdote.

D'autres exemples de l'emploi du mot Negre relèvent d'une représentation de l'organisation raciale dans laquelle a vécu Léonora et marquent les différences socio-historiques entre les Noirs, les Blancs et 
les Indiens ${ }^{8}$. La traduction de chaque instance du mot Nègre par black atténue ces contrastes et prête à Léonora une conscience qui n'est pas la sienne. C'est en parlant qu'elle commence de plus en plus à prendre conscience d'elle-même et qu'elle se met à commenter son propre récit. Les circonstances de sa vie l'empêchent pourtant de se voir et de se dire autre que Nègre. Bien que l'histoire du combat mené par les Noirs des États-Unis pour l'égalité et les droits civiques ait touché et influencé de nombreux écrivains antillais, elle n'a aucune place dans l'histoire de Léonora. La sensibilité du public américain devrait-elle done être protégée lorsqu'en Guadeloupe, parmi les populations créoles illettrées, une telle sensibilité n'a été amorcée qu'au cours des vingt dernières années?

Dans deux des exemples placés en appendice (2 et 3), il y a lieu de s'interroger sur la valeur éthique de l'utilisation généralisée du terme black pour désigner ce qui pour Léonora représente une identité et une auto-représentation charriée par l'histoire. Le premier exemple fait référence au régime de Vichy imposé en Guadeloupe par le gouverneur Sorin et illustre la ténacité des conséquences psychiques et sociales de l'esclavage. Le second relate les réflexions de Léonora sur les différences perçues entre Nègres et Blancs, en particulier en matières conjugales. Être Nègre, ce n'est pas seulement avoir la peau noire, c'est porter en soi les marques de quatre siècles d'injustice, c'est avoir assimilé le mépris dont on a été l'objet et c'est se retrouver invisible, absent des livres d'école et de l'histoire officielle. Ce ne sont pas des Noirs qui ont été entassés comme du bétail sur les négriers, mais bien des Nègres, et ce ne sont pas des Noirs qui se détestent eux-mêmes au point de refuser de parler leur langue maternelle, le créole, mais bien des Nègres.

Je veux suggérer par là que, afin de préserver la superposition des couches représentatives dans le discours de Léonora, il aurait été préférable de recourir à plusieurs stratégies différenciées selon les instances. Pourquoi ne pas retenir le terme français, par exemple, ou revenir au terme créole, lorsqu'il est clairement avisé de ne pas atténuer les propos de Léonora ou de maintenir le contraste entre l'identité et la

\footnotetext{
${ }^{8}$ Au lendemain de l'esclavage, il s'est avéré nécessaire d'attirer une nouvelle main-d'œuvre dans les colonies afin de maintenir le taux de production des plantations. Plus de 40000 travailleurs sont ainsi venus de l'Inde sous contrat limitê mais très peu ont été rapatriés (voir Bébel-Gisler, 1985a, p. 304, n. 11).
} 
couleur? Le terme Nègre n'étant jamais employé en tant qu'insulte directe, le recours aux termes nigger et negro n'est certes pas nécessaire, en tout cas dans le sens où ils sont subtilement différenciés, par exemple, pour traduire l'incipit d'un essai de Fanon, intitulé «L'expérience vécue du Noir", et commenté par le théoricien postcolonial Homi Bhabha : " "Sale nègre!" ou simplement : "Tiens, un nègre!"v qui donne en anglais : “"Dirty nigger!” Or simply, "Look, a Negro!"» (1994, p. 236). La seule occurrence que j'aie relevée du terme Negro est donc pour le moins incongrue : "D'ailleurs, dans la communauté, les hommes sont rares comme des Nègres aux yeux bleusw (1985a, p. 266) traduit par *Besides, men are as scarce in the congregation as blue-eyed Negroes" (1994, p. 209). L'emploi du terme créole dans les deux traductions, tel que l'illustre l'exemple suivant, me semble offrir une solution idéale dans plusieurs cas :

Sa famille raconte qu'on lui a jeté un sort, qu'un "gwonèg nouèr qu'elle avait dédaigné a envoyé un esprit sur elle... (1985a, p. 237).

Her family tells how a spell was put on her, that a gwonèg nouè she once snubbed sent a spirit to work on her... $(1994$, p. 186).

Bien que cette expression relève ici du discours rapporté, elle aurait pu servir à traduire le malaise que ressentait Léonora dans le contexte urbain de Pointe-à-Pitre et qui est suggéré dans la proposition suivante : «devant la boutique de M. Fostin, un gros Nègre tout noir [...]" (1985a, p. 96) traduit par : «in front of Monsieur Fostin's store. He was a large, dark, black man $[\ldots]$ » (1994, p. 73). La même stratégie aurait aussi empêché la confusion entre ce gros Nègre tout noir, décrit dans l'exemple précédent, et le grand Nègre noir que, quelques pages plus loin, Léonora trouve séduisant :

Ce jour-là, une amie m'accompagnait, et lui était avec un grand Nègre noir qu'il faisait passer pour son frère (1985a, p. 115).

That day, a girlfriend had accompanied me, and he was with a big, dark black man he passed off as his brother (1994, p. 87).

La nuance est subtile entre ces deux instances mais, dans le premier cas, l'adjectif tout place l'expression française dans un registre populaire, soit l'équivalent de l'expression créole, traduisant ainsi la peur inspirée par la 
noirceur de l'homme alors que, dans le deuxième cas, il ne s'agit que de la description d'un grand homme à la peau noire. En anglais, au lieu de la différence banale entre les adjectifs big et large, cette distinction aurait pu être maintenue par l'emploi du terme créole dans le premier cas et la solution adoptée dans le second.

\section{L'invisibilité du créole}

Une autre manifestation de la perte du ton militant de Léonora lorsqu'elle parle anglais se trouve dans le chapitre $X$, intitule «Le mariage? Une remorque de misères, un baril plein de tessons de bouteillesw. Le passage suivant, en particulier l'expression le bâton se met à parler français, préfigure le chapitre XIII, intitulé "L'amour du français, une maladie qui rend fou" :

-La ou sòti la, sé voyé ou ban mwen.

Là d'où tu viens, on ne voulait plus de toí, on t'a renvoyé vers moi. Cette petite phrase, les hommes ne peuvent la supporter. Le ad'où tu viensı suffit à déchaîner l'orage, le bấton se met à parler français et les tôles à chanter (1985a, p. 192).

-La ou sòti la, sé voyé ou ban mwen.

Where you've come from they don't want you anymore so they've sent you back knocking on my door.

Men can't stand this little jingle. The "Where you've come from" suffices to bring on the storm, to start the rod ringing and the tin roof singing (1994, p. 150).

La violence perpétrée sur les Guadeloupéens par l'imposition de la langue française équivaut, dans l'analyse remarquablement subtile conduite par Léonora, à la violence faite aux femmes à l'intérieur de l'institution du mariage. Le français et le mariage figurent parmi les instruments du colonialisme qui passent pour des véhicules de salut social et religieux. Il s'agit donc ici d'une perte plus considérable que les pertes plus ou moins anodines qui sont l'effet de toute traduction. $\mathrm{La}$ traductrice aurait pu ajouter une note à cet effet ou même faire chanter les toles en français.

La demière difficulté qui retiendra mon attention concerne la traduction d'un terme créole qui, par le truchement de deux traductions successives, perd de son importance jusqu'à être rendu invisible. Il s'agit 
du lexème $k \grave{~ q u i, ~ " c o r r e s p o n d a n t ~ d e ~ c o r p s ~ e n ~ f r a n c ̧ a i s ", ~ s e l o n ~ u n e ~ l o n g u e ~}$ note de Dany Bébel-Gisler, "désigne la personne tout entière, le moi" (1985a, p. 302, n. 5). L'importance de ce terme réside dans le fait qu'il permet d'évoquer

en même temps l'ensemble des rapports qui lient l'individu à sa famille, aux vivants comme aux morts, en une chaîne symbolique qui intègre les rapports à la maladie, à la vie, à la mort, aux ancêtres, aux esprits, à la nature. Rapports noués au sein de la violence esclavagiste, porteurs de cette histoire inscrite dans le corps et dans la langue créole où gît la mémoire d'un monde imposé et subi, mais combattu et subverti par l'imaginaire et le symbolique (1985a, p. 303).

Dany Bébel-Gisler relate dans Le Défi culturel guadeloupéen quelques anecdotes qui aident à mieux comprendre la richesse de ce lexème, la difficulté de sa traduction et les conséquences sociales des conditions diglossiques dans lesquelles il est utilisé. Elle raconte, en particulier, l'épisode qui suit :

Une femme à qui un psychologue demandait des nouvelles de son fils qu'il suivait, lui répondit : aJe lui laisse battre son corps", transposant directement en français aan ka lésé-i bat kò $a-i *$, je ne me fais pas de bile, je le laisse se débrouiller. Le psychologue français conclut à de l'autopunition masochiste et demanda une sanction contre la mère (1989, p. 159).

Dans Léonora donc, il est important de se souvenir, en l'absence des termes créoles, que le mot corps désigne la personne tout entière qui, dans la philosophie créole, n'est jamais divisible en corps et âme. La traductrice ajoute à la note métalinguistique de Bébel-Gisler qu'elle se sert du terme self ou de celui plus littéral de body pour rendre la notion de $k \grave{o}$ (1994, p. 238, n. 10). Cette note apparaît au début du livre et, pour les raisons avancées plus haut, il est raisonnable de s'attendre que la lectrice ait perdu de vue les subtilités associées à ce terme. Mon analyse comparative, d'ailleurs loin d'être complète, révèle que cette solution, bien que paraissant tout à fait acceptable dans la note, s'avère problématique dans le texte. La traduction littérale de corps par body rend parfaitement bien la notion de $k \grave{o}$ telle qu'elle a été expliquée et tel que l'atteste l'exemple suivant : «La France possède notre corps, c'est elle qui mène notre vie, pas nous" (1985a, p. 258) traduit par : «France owns our 
bodies, and it's France that directs our lives, not us" (1994, p. 203). La même solution est aussi heureuse dans un passage tiré de l'épilogue :

Ne faut-il pas d'abord être, être un homme, une femme, une personne? Récupérer ce corps volé par le maître, agressé par la France assimilatrice? (1985a, p. 298)

Mustn't one first exist, be a man, a woman, a person? Reclaim this body stolen by the master, violated by France the assimilator? (1994, p. 234)

Par contre, en ce qui concerne l'emploi de self, la solution est moins heureuse à cause de la nature pronominale du terme qui, dans les deux extraits suivants, en dissout totalement la proéminence sémantique. Une fois de plus, la voix de Léonora, qui déplore la perte moderne de l'esprit communautaire, s'adoucit en anglais :

Rien en communauté, aucun partage. Leur corps, leurs amis, leur famille, c'est leur maison, leurs meubles (1985a, p. 254).

Nothing is shared, nothing done together as neighbors. What counts is themselves, their friends, their families, their house, their furniture (1994, p. 199).

Cette traduction change aussi le sens de la phrase en créant une seule série avec les termes des deux côtés de l'équation. Léonora déplore en fait la réification du corps, des amis et de la famille, leur importance ayant été supplantée par les biens matériels qui transforment une vie communautaire en existence individualiste. Dans le second exemple, l'impact profondément philosophique des termes créoles se trouve banalisé en anglais :

Sortir des pattes du colon, d'accord, mais ne pas lâcher notre corps dans n'importe quoi (1985a, p. 259).

It's fine to escape from under the thumb of the colonizers, but not simply to give ourselves over to the next thing that comes along (1994, p. 203). 


\section{Conclusion}

Au cours de son dialogue avec Dany, Léonora se traduit elle-même. En tant que narratrice fictive de son propre récit, elle est mise en scène par Dany comme signe de sa propre identité et le lien ainsi créé entre les deux femmes assure la poursuite dynamique de cette identité. L'encadrement différent qui est donné à Léonora dans sa version anglaise l'immobilise sur scène. Elle a été relue, réinterprétée et re-présentée par un nouvel appareil universitaire qui, d'un côté, s'efface volontairement pour laisser paraître le personnage fictif et, d'un autre côté, privilégie la valeur ethnographique, donc réifiée, de ce personnage. L'entreprise de Dany Bébel-Gisler se frayait une voie difficile entre les langues et les genres. Les auteures de l'article critique qui vient clore l'édition américaine font remarquer qu'au départ, le projet de la sociologue constituait aussi un projet universitaire et anthropologique mais que, comme souvent dans le cas de recherches menant à la création du genre du testimonio, l'investissement personnel de la chercheuse la pousse à s'inscrire ellemême dans le récit, parce qu'elle est elle-même Guadeloupéenne, parce que sa mère était aussi travailleuse de canne et parce qu'elle est engagée politiquement dans la réalité de la Guadeloupe. Cette présence discursive est ressentie comme le ressort mutuel d'une dynamique identitaire qui fait de Léonora une ceuvre ouverte. Dany et Léonora partent toutes deux à la découverte d'elles-mêmes et, ensemble, font part d'une expérience collective qui demeure inachevée. Dany insiste là-dessus :

Jamais, en écoutant Léonora, je ne me suis sentie étrangère, absente de ses interrogations, de ses doutes, de ses recherches. Une grande part de ce qu'elle me disait répondait en moi, résonnait dans mon corps, faisait resurgir des sensations oubliées. (1985a, p. 298)

Ce lien dialogique qui unit les deux femmes est fragile et survit difficilement à ul'épreuve de l'étrangern (Berman, 1984). En réponse à ma propre question, En anglais, Léonora parle-t-elle encore et à qui?, je réponds donc oui, elle parle encore mais on ne sait plus très bien à qui elle s'adresse. En tant qu'agent culturel, Léonora ne se réduit pas facilement au silence mais la présence de Dany, en revanche, est compromise et déplacée. La traductrice américaine ne vient pas non plus s'interposer pour remplir ce rôle nécessaire d'interlocutrice intéressée, ce qui a pour conséquence de diminuer considérablement la force illocutoire et perlocutoire de la narration de Léonora et de briser l'élan herméneutique 
que les conditions d'énonciation créées par Dany Bébel-Gisler lui avait permis d'entreprendre. Les quelques défauts relevés ici ne sont pas, d'un point de vue linguistique, de grands défauts et leur correction serait relativement aisée. En particulier, l'ajout d'une préface de la main de la traductrice aurait pu faire part à la lectrice de la perspective dans laquelle elle a lu, interprété et traduit $L$ 'histoire enfouie de la Guadeloupe telle que Léonora la raconte. Étant donné la visée didactique de cette traduction, il est indispensable d'attirer l'attention du public sur le fait que toute traduction signifie forcément appropriation mais qu'elle peut et doit se faire avec le moins de violence possible.

University of Alberta

\section{Références}

ARNOLD, A. James (1992). «Poétique forcée et identité dans la littérature des Antilles francophones". L'Héritage de Caliban, éd. Maryse Condé. Pointe-à-Pitre, Éditions Jasor, pp. 19-27.

BAKHTINE, Mikhail (1978). Esthétique et théorie du roman. Trad. Daria Olivier. Paris, Gallimard.

BÉBEL-GISLER, Dany (1976). La langue créole, force jugulee. Paris, L'Harmattan.

Seghers.

(1985a). Léonora: l'histoire enfouie de la Guadeloupe. Paris,

(1985b). Les enfants de la Guadeloupe. Paris, L'Harmattan.

(1989). Le Défi culturel guadeloupéen : devenir ce que nous sommes. Paris, Éditions Caribéennes.

(1994). Leonora: The Buried Story of Guadeloupe. Trans. Andrea Leskes. Charlottesville and London, University Press of Virginia.

BERMAN, Antoine (1984). L'éprenve de l'étranger. Paris, Gallimard. 
BHABHA, Homi K. (1994). The Location of Culture. New York, Routledge.

BHÊLY-QUÉNUM, Olympe (1988). Snares Without End. Trans. Dorothy S. Blair. Charlottesville and London, University Press of Virginia.

BOYCE DAVIES, Carole and Elaine SAVORY FIDO, eds. (1990). Out of the Kumbla. Trenton, NJ, African World Press, Inc.

CERVONI, Jean (1987). L'énonciation. Paris, Presses Universitaires de France.

CÉSAIRE, Aimé (1990). Lyric and Dramatic Poetry 1946-82. Trans. Clayton Eshleman and Annette Smith. Charlottesville and London, University Press of Virginia.

CHARAUDEAU, Patrick (1983). Langage et Discours. Paris, Hachette.

CONDE, Maryse (1992). I, Tituba, Black Witch of Salem. Trans. Richard Philcox. Charlottesville and London, University Press of Virginia.

DANON-BOILEAU, Laurent (1987). Le sujet de l'énonciation : psychanalyse et linguistique. Paris, Ophrys.

DEPESTRE, Rene (1990). The Festival of the Greasy Pole. Trans. Carrol F. Coates. Charlottesville and London, University Press of Virginia.

DJEBAR, Assia (1992). Women of Algiers in Their Apartment. Trans. Marjolijn de Jager. Charlottesville and London, University Press of Virginia.

DUCROT, Oswald (1980, 1972). Dire et ne pas dire. Paris, Hermann. (1984). Le dire et le dit. Paris, Les Éditions de Minuit.

FANTOURÉ, Alioum (1989). Tropical Circle. Trans. Dorothy S. Blair. Charlottesville and London, University Press of Virginia. 
FOLKART, Barbara (1991). Le conflit des énonciations : traduction et discours rapporté. Candiac, Québec, Les Éditions Balzac.

GLISSANT, Édouard (1989). Caribbean Discourse : Selected Essays. Trans. J. Michael Dash. Charlottesville and London, University Press of Virginia.

JUMINER, Bertène (1989). The Bastards. Trans. Keith Q. Wamer. Charlottesville and London, University Press of Virginia.

KATEB, Yacine (1991), Nedjma. Trans. Richard Howard. Charlottesville and London, University Press of Virginia.

KERBRAT-ORECCHIONI, Catherine (1980). L'énonciation : de la subjectivité dans le langage. Paris, Armand Colin.

KUTZINSKI, Vera $M$. et Cynthia MESH-FERGUSON (1995). "Convergences». Afterword, Bébel-Gisler (1995), pp. 261-268.

MALENA, Anne (1995). "Léonora : auteure d'elle-même", Authorship/Authority : Proceedings of the Fifh Annual Graduate Conference in French, Francophone, and Comparative Literature, Columbia University, March 3-4 1995, eds. Vincent Desroches and Geoffrey Tumovsky. New York, FGSU, pp. 138-144.

MAXIMIN, Daniel (1989). Lone Sun. Charlottesville and London, University Press of Virginia.

NIRANJANA, Tejaswini (1992). Siting Translation : History, PostStructuralism, and the Colonial Context. Berkeley, Los Angeles, and Oxford, University of Califomia Press.

OYÔNÔ-MBIA, Guillaume and Seydou BADIAN (1988). Faces of African Independence : Three Plays. Trans. Richard Bjornson. Charlottesville and London, University Press of Virginia.

PATERSON, Janet M. (1993). Moments postmodernes dans le roman québécois. Ottawa, Presses de l'Université d'Ottawa, $1^{\text {tre }}$ éd. 1990. 
SENGHOR, Léopold Sédar (1991). The Collected Poetry. Trans. Melvin Dixon. Charlottesville and London, University Press of Virginia.

U TAM'SI, Tchicaya (1989). The Madman and the Medusa. Trans. Sonja Haussmann Smith and William Jay Smith. Charlottesville and London, University Press of Virginia.

\section{Appendice}

\section{Exemple 1:}

MWEN SÉ GWADLOUPÉYEN

Mwen sé timoun enkyèt a on litèt inkyèt.

On ti lilèt ki vwè parèt é disparèt

- syèk dèyè syèk -

disparèt é parèt

fanm é nonm zòt senné kon ban pisyèt

[...]

Apré sa

kijan fê ou vlé fê mwen kwè

mwen sé vou

vou sé mwen?

Mwen tala!

Sonny Rupaire

Mwen sé Gwadloupéyen

Mwen sé timoun enkyèt a on lilèt inkyèt.

On ti lilèt ki vwè parèt é disparèt

- syèk dèyè syèk -

disparèt é parèt

\section{MOI, GUADELOUPÉEN}

Moi, je suis l'enfant inquiet d'une petite île inquiète.

Une petite lle qui a vu paraitre et disparaitre

- siècle après siècle -

disparaître et apparaître des hommes et des femmes que vous avez péché à la senne tel un ban d'alevins,

Après cela

comment voulez-vous me faire croire que moi c'est vous, que vous c'est mot? Moi, cet enfant-là! (traduction D. Bébel-Gisler)

I am Guadeloupean I am a troubled child from a small troubled island.

A small island that has seen appear and disappear - century after century disappear and appear 
fanm é nonm zòt senné kon ban pisyèt

[...]

Apré sa

kijan fề ou vlé fè mwen kwè

mwen sé vou

vou sé mwen?

Mwen tala! women and men that you caught in your net as if they were a school of fish,

After that

how can you make me believe

that I am you, that you are me?

I, that child!

Sonny Rupaire

\section{Exemple 2:}

Alors, disent les anciens, il nous faudrait un homme fort comme Sorin pour faire marcher le pays. Nous, Nègres, ne marchons que si on nous fouette. Les Nègres sont mauvais. Vous demandez l'indépendance? Les Nègres vont commander aux Nègres alors? Si le Nègre ne sent pas le fouet sur sa peau, il ne fait rien. Paroles que tout ça. N'empêche qu'on les entend souvent ici. Paroles contre nous-mêmes. A la moindre anicroche, c'est la faute du Nègre. Le Nègre méprise le Nègre. Je ne suis pas d'accord avec ces paroles de dédain, de mépris pour le Nègre, ton frère. Ces gens en sont toujours à l'esclavage. Les Blancs, c'est vrai ont fait marcher les Nègres à coups de fouet. C'est de l'histoire. Les Blancs aussi ont leur histoire, mais elle n'apparaît pas comme celle des Nègres. Ce que nous devons rechercher : l'accord entre nous. Alors, nous pourrons vivre bien, normalement. En temps Sorin, rien de normal (p. 126).

So, the old ones say, we need a strong man like Sorin to keep the country running. We blacks don't move unless we're whipped. The blacks are bad. You want independence? So blacks will lead blacks? If black people don't feel the whip on their skins, they won't work. Only words, all that. Yet we hear them repeated often here. Words against ourselves. The least snag and it's the black man's fault. Blacks scom blacks. I don't agree with these disdainful words, this scomful judgment of blacks, of your brother. Those people act as if we're still slaves. Yes, it's true, the whites made the blacks jump by whipping them. It's part of our history. The whites too have their history, but it seems different from ours. What we must search 
for is agreement among ourselves. Then we could live well, live normally. Under Sorin, nothing was normal (p. 96).

\section{Exemple 3 :}

Certains racontent que chez les Blancs ça ne se passe pas comme dans les ménages de Nègres. Ce sont des bêtises. Les gens ne réfléchissent pas et disent n'importe quoi. J'ai vu, à Prise-d'Eau, un monsieur blanc foutre des coups à sa femme blanche. Alors, pourquoj toujours affirmer que les Blancs sont ci, les Nègres sont ça? Toujours Nègres, Nègres, Nègres... Les Nègres ne sont pas les plus mauvais. Si on regarde bien, toutes les catégories d'hommes se disputent et se bagarrent. Ce n'est pas une affaire de race. Blanc ou noir, un homme est un homme, et chaque homme a un comportement à lui (p. 191).

Some people say that it's different with white people, that things don't happen the way they do in the household of blacks. That's stupid. They don't think, they say whatever comes into their heads. At Prise-d'Eau I saw a white man beat his white wife. So why always say that the whites are like this, the blacks like that? Always the blacks, the blacks, the blacks... the blacks aren't the worst. If you look carefully, all groups of men argue and fight. It's not a question of race. White or black, a man is a man, and each man has his own particular way of behaving (p. 149).

RÉSUMÉ : En anglais, Léonora parle-t-elle encore et à qui? - Dany Bébel-Gisler, sociolinguiste et attachée de recherche au C.N.R.S., a écrit Léonora : l'histoire enfouie de la Guadeloupe afin de célébrer la culture de son île natale et de produire un contre-discours au discours national français. $\dot{A}$ partir d'une série d'entrevues en créole avec Léonora, travailleuse de canne née en 1919, Dany établit le texte d'un romantémoignage dans lequel elle maintient certains passages en créole. Bien que Bébel-Gisler s'approprie la voix de Léonora, elle le fait dans un but didactique et militant et cet acte littéraire de traduction peut être défendu comme un geste de solidarité visant à explorer et à faire connaître le pouvoir de résistance des pratiques culturelles de la Guadeloupe. Le présent article analyse la version américaine de Léonora traduite par Andrea Leskes, publiée par «University Press of Virginia», afin de déterminer si elle parvient à renouveler ce geste de solidarité. Une question bakhtinienne (Qui parle?) est posée et trois problèmes spécifiques sont examinés pour tenter d'y répondre : l'emploi du créole, 
la relation dialogique entre le créole et le français et la valeur de contrediscours du roman.

ABSTRACT : In English, is Léonora Still Speaking and to Whom? Dany Bébel-Gisler, a sociolinguist and member of the C.N.R.S, wrote Léonora : The Buried Story of Guadeloupe as a celebration of the culture of her native island and as counter-discourse to the French national discourse. Basing her text on a series of interviews conducted in Creole with Léonora, a cane worker born in 1919, Dany produces a novel in the genre of the testimonio in which she maintains many passages in Creole. Even though Bébel-Gisler appropriates Léonora's voice, her goal is didactic and militant and she accomplishes a literary act of translation that can be defended as a gesture of solidarity meant to explore and promote the cultural practices of Guadeloupe as a powerful force of resistance. This article examines the American version of Léonora translated by Andrea Leskes, published by University Press of Virginia, in order to determine whether this gesture of solidarity is renewed in the translation. In attempting to answer the Bakhtinian question, "who is speaking?", three particular problems are treated : the use of Creole, the dialogical link between Creole and French, and the counter-discursive nature of the novel.

Anne Malena : Modern Languages and Comparative Studies, University of Alberta, 200 Arts Building, Edmonton, AB T6G 2E6, courriel : amalena@gpu.srv.ualberta.ca 